\title{
CIRUGÍA ENDOSCÓPICA EN UN NIÑO CON ATRESIA UNILATERAL DE COANA
}

\section{Endoscopic surgery in a child with unilateral choanal atresia}

\author{
Diego HELLÍN-MESEGUER ${ }^{1}$; Pablo MELGAREJO-MORENO*1; Carmen ÁLVAREZ- \\ SANTACRUZ ${ }^{2}$; Fares GHANI $^{2}$; Javier GALINDO-ORTEGO ${ }^{2}$ \\ ${ }^{1}$ Universidad Católica de Murcia. Hospital Universitario Reina Sofía. Servicio de Otorrinolaringología. Murcia. España. \\ ${ }^{2}$ Hospital Universitario Santa María. Servicio de Otorrinolaringología. Lleida. España \\ *Correspondencia: pmelgarejo3@gmail.com
}

Fecha de recepción: 26 de octubre de 2015

Fecha de aceptación: 14 de noviembre de 2015

Fecha de Publicación: 15 de enero de 2016

Conflicto de intereses: Los autores declaran no tener conflictos de intereses

Imágenes: Los autores declaran haber obtenido las imágenes con el permiso de los pacientes

Política de derechos y autoarchivo: se permite el autoarchivo de la versión post-print (SHERPA/RoMEO)

Licencia CC BY-NC-ND. Licencia Creative Commons Atribución-NoComercial-SinDerivar 4.0 Internacional

(C) Universidad de Salamanca. Su comercialización está sujeta al permiso del editor

Introducción y objetivo: La atresia de coanas es infrecuente, siendo dos veces más frecuente en mujeres que en varones, y en el $50 \%$ de los casos aproximadamente se asocia a otras anomalías congénitas. Aunque ha existido cierta controversia sobre el tratamiento de elección de esta malformación en la última década, la introducción de los endoscopios en la cirugía nasosinusal ha mejorado los resultados. Descripción del caso: Presentamos el caso de un niño que presenta rinorrea crónica izquierda acompañada de obstrucción nasal persistente. Mediante endoscopia y TAC se confirma el diagnóstico de atresia de coana izquierda. El tratamiento de la atresia de coana unilateral se realiza mediante cirugía endoscopia con creación de colgajos de mucosa intranasales, evitando el taponamiento nasal. Discusión: Diferentes técnicas han sido consideradas para el tratamiento de la atresia de coanas, que incluyen las de abordaje tradicional y las endoscópicas. Los resultados de la cirugía endoscópica en la atresia de coanas varían según los diferentes autores. Los dos aspectos más importantes en estos resultados en niños son la creación de colgajos de mucosa y evitar el taponamiento nasal. Conclusiones: El tratamiento quirúrgico de la atresia de coana unilateral mediante cirugía endoscopia puede reducir la posibilidad de reestenosis.

PALABRAS CLAVE Atresia coanas; cirugía endoscópica; edad pediátrica; Tomografía computarizada; obstrucción nasal

SUMMARY

Introduction and objective: Choanal atresia is rare, being twice as common in women than in men, and in $50 \%$ of cases approximately associated with other congenital anomalies. Although there has been some controvesia about the treatment of choice of this malformation in the last 
decade, the introduction of endoscopes in sinus surgery has improved outcomes. Case description: We report the case of a child who has left chronic rhinorrhea accompanied by persistent nasal obstruction. By endoscopy and CT diagnosis of left choanal atresia is confirmed. Treating choanal atresia unilateral endoscopic surgery is performed by creating flaps with intranasal mucosa, preventing nasal packing. Discussion: Different techniques have been considered for the treatment of choanal atresia, which include traditional approach and endoscopic. The results of endoscopic surgery in the choanal atresia vary according to the different authors. The two most important aspects of these results are creating flaps nasal mucosa and to avoid placing nasal packing. Conclusions: Surgical treatment of unilateral choanal atresia endoscopic surgery can reduce the risk of restenosis.

\section{INTRODUCCIÓN}

La atresia de coanas congénita fue descrita por primera vez por Roederer en 1755 [1] y el primer tratamiento quirúrgico se realizó en 1851 [2]. Aunque ha existido cierta controversia sobre el tratamiento de elección de esta malformación en la última década, la introducción de los endoscopios en la cirugía nasosinusal ha mejorado los resultados [3]. La atresia de coanas es infrecuente, ocurre aproximadamente en 1 de cada 7000-8000 nacimientos [4]. Es dos veces más frecuente en mujeres que en varones, y en el $50 \%$ de los casos aproximadamente se asocia a otras anomalías congénitas. Aunque la atresia de coanas unilateral es más común que la atresia de coanas bilateral (65 a 75\%), la bilateral debe tratarse de forma urgente puesto que provoca insuficiencia respiratoria en el recién nacido. La atresia de coanas bilateral es una urgencia médica, pero no una urgencia quirúrgica. La atresia de coanas se asocia con otras anomalías congénitas conocidas como CHARGE que incluye colobomas, malformaciones cardiacas, retraso mental y del crecimiento, anomalías genitourinarias y óticas. También se ha observado junto a la atresia de coanas polidactilia, síndrome de Crouzon, craneosinostosis, microencéfalo, meningocele, asimetría facial e hipertelorismo $[5,6]$.

\section{DESCRIPCIÓN}

Niño de 9 años que presentaba rinorrea crónica izquierda y bloqueo permanente de la fosa nasal izquierda. Mediante endoscopia nasal y TC de senos paranasales se confirmó el diagnóstico de atresia de coana izquierda (Figura 1).

Se realizó una incisión en la mucosa sobre la placa atresia con ayuda de un endoscopio de $0^{\circ}$ y $2,7 \mathrm{~mm}$, elevando un colgajo de charnela superolateral. En la misma fosa, en la unión de los dos tercios anteriores con el tercio posterior del septum, se practicó incisión vertical en la mucosa septal desde una altura marcada por el nivel inferior de la cola del cornete medio hasta el suelo de la fosa.

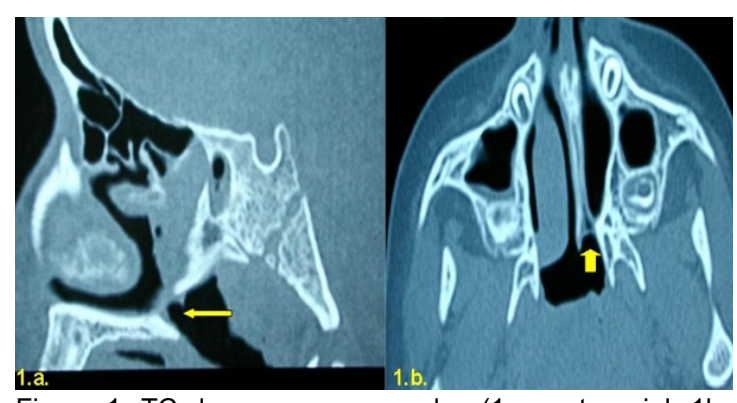

Figura 1. TC de senos paranasales (1a. corte axial. 1b. corte sagital). Las flechas indican la zona de la atresia.

Desde los extremos de esta incisión se iniciaban dos incisiones horizontales y paralelas hasta el fondo de la fosa, resecando este colgajo de mucosa (Figura 2). Posteriormente se resecó el hueso tras retirar la mucosa y abrir un túnel superior derecho posterior desde la fosa nasal izquierda. Este hueso se fue extirpado cuidadosamente con la pinza de Blakesley y gubia para no romper la mucosa septal del lado derecho. A través del túnel derecho se llegó al final del vómer y se retiró definitivamente todo el hueso marcado y la mucosa adyacente pudiendo ver la gasa que habíamos colocado en cávum (Figura 2).

En la mucosa del lado derecho del tercio posterior del tabique se hicieron dos incisiones horizontales para diseñar un colgajo que se replegara hacia delante en la fosa izquierda para ampliar anteriormente la rinofaringe $y$ cubrir la parte ósea posterior del septum en la fosa izquierda (Figura 3). También se dejó un pequeño colgajo mucoso sobre los restos laterales de la placa de atresia para cubrir los bordes cruentos de la atresia y evitar sinequias (Figura 3). 
Al final de la intervención se colocó una lámina de silastic estrecha rodeando todo el tabique y asomando por ambas fosas uniéndolo con un punto fuera de la fosa nasal protegiendo la columela con un cilindro de gasa.

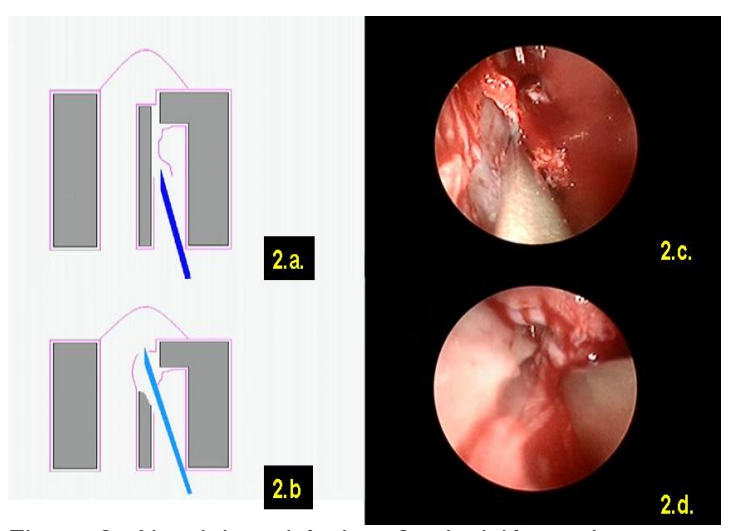

Figura 2. Abordaje quirúrgico. 2a: incisión en la mucosa septal anterior a la atresia. 2b: punto de incisión de la mucosa posterior a la atresia. $2 \mathrm{c}$ : imagen endoscópica. $2 \mathrm{~d}$ : imagen endoscópica de la incisión.

A los cinco días se retiró el silastic, presentando fisuras en ambos vestíbulos por contacto. Al séptimo día se realizó nueva cura con endoscopio de 2,7 $\mathrm{mm}$ retirando costras y restos de coágulos, tras la cura se colocó pomada con Diprogenta ${ }^{\circledR}$ y hialurónico en ambas fosas nasales.

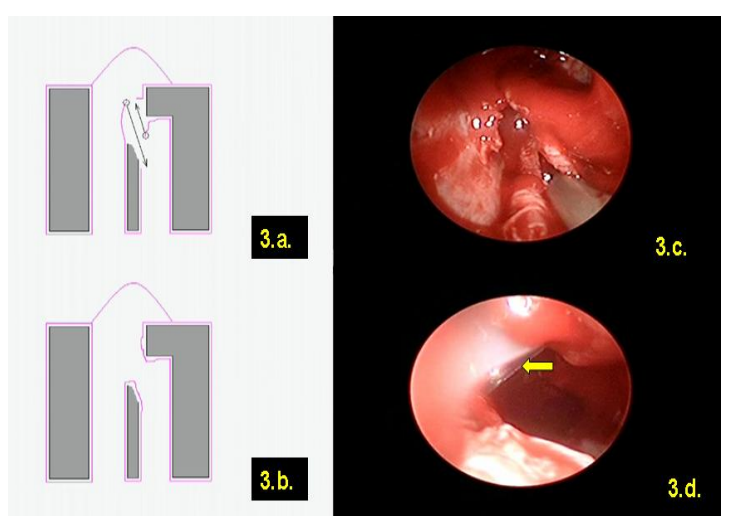

Figura 3. 3a: colocación del colgajo de mucosa hacia adelante para cubrir la incisión en el septum. 3c: imagen endoscópica. 3c: colocación del otro borde de mucosa para cubrir la zona cruenta en el borde de la placa de atresia. 3d: imagen por endoscopia.

En la revisión a los 6 meses el niño estaba asintomático y la ventilación nasal evaluada con espejo de Glatzell era simétrica en ambas fosas nasales. No se realizó endoscopia nasal por no considerarla imprescindible, debido a lo mal que lo paso el niño con las curas postoperatorias.

\section{DISCUSIÓN}

Desde 1910 se ha seguido la clasificación de Fraser [7] que establecía que la obstrucción podía deberse en un $90 \%$ ósea y en un $10 \%$ membranosa. Recientes estudios mediante tomografía computarizada han determinado que la obstrucción es en un $70 \%$ mixta óseamembranosa y en un $30 \%$ ósea [8].

Diferentes técnicas han sido consideradas para el tratamiento de la atresia de coanas que incluyen las de abordaje tradicional y las endoscópicas. Dentro de las tradicionales se encuentran la punción intranasal, la reparación transpalatina y el abordaje transeptal sublabial [9-11].

El abordaje transeptal sublabial es útil especialmente en neonatos con otras malformaciones craneofaciales o intranasales [12]. En estos casos, tal como ha sido descrito por primera vez por Stankiewiez, los endoscopios pueden ser utilizados tras elevar la mucosa del suelo de la fosa y el pericondrio del septum [13].

Schoem ha recomendado evitar el taponamiento nasal tras la cirugía endoscópica de la atresia de coanas [14]. La resección cuidadosa de la porción posterior del septum reduce la necesidad de proteger la mucosa para evitar la formación de tejido de granulación y sinarquías. Van Den Abbeele et al. [15] han utilizado en sus pacientes el taponamiento nasal durante solo 2 días tras la cirugía y según sus conclusiones el taponamiento nasal no es necesario.

Los resultados de la cirugía endoscopia en la atresia de coanas van desde un 75 a un $85 \%$ de éxitos según los diferentes autores [16, 17]. Los dos aspectos más importantes en estos resultados son la creación de colgajos de mucosa y evitar el taponamiento nasal [18]. El uso de colgajos está basado en que solo una capa epitelial es capaz de prevenir la retracción en la cicatrización mientras que el taponamiento provoca ulceración, favorece la infección y daña los tejidos sanos [19-21].

\section{CONCLUSIONES}

El tratamiento quirúrgico de la atresia de coana mediante cirugía endoscópica con creación de colgajos de mucosa intranasales y evitando el taponamiento nasal, puede reducir la posibilidad de reestenosis. 


\section{BIBLIOGRAFÍA}

1. Otto W. Lehrbuch der pathologie, Anatomy des Menchen und der Thiere. Vol I. Berlin, Alemania. A Ruecker, 1830. pp. 180-3.

2. Emmert C. Steneochorie und atresia der choannen. En Lehrbuch der Speciellen Chirurgie (Vol. 2). Stuttgart, Alemania. Dann, 1954. pp. 535-8.

3. Metin Önerci T, Taskin Yücel Ö, Ögretmenoglu $\mathrm{O}$. Transnasal endoscopic surgery in choanal atresia. Operative Techniques in Otolaryngology 2006;17:143-6.

4. Hengerer AS, Newburg J. Congenital malformations of the nose ad paranasal sinuses. En Bluestone CD, Stool SE (Eds): Pedriatric Otolaryngology (Ed). Filadelfia, Saunders, 1990. pp. 727-8.

5. Morgan DW, Bailey CM. Current management of choanal atresia. Int J Pediatr Otorhinolaryngol 1990;19:1-13.

6. Kaplan LC. The CHARGE association: choanal atresia ad multiple congenital anomalies. Otolaryngol Clin North Am 1989;22:661-72.

7. Fraser JS. Congenital atresia of the choane. Br J Med 1910;2:1698-701.

8. Brown OE, Powenell P, Manning SC. Choanal atresia. A new anatomic classification ad clinical management applications. Laryngoscope 1996;106:97-101.

9. Carpenter RJ, Neel HB. III: Correction of congenital choanal atresia in children and in adults. Laryngoscope 1977;87:1304:11

10. Owens $\mathrm{H}$. Observations in treating twenty-five cases of choanal atresia by transpalatine approach. Laryngoscope 1965;75:84-104.

11. Park HA, Brockenbrough J, Stankiewicz J. Endoscopic versus traditional approaches to choanal atresia. Otolaryngol Clin North Am 2000;33:77-90.
12. Krespi YP, Husain S, Levine TM, Reede DL. Sublabial transseptal repair of choanal atresia or stenosis. Laryngoscope. 1987;97:1402-6.

13. Stankiewicz JA. The endoscopic repair of choanal atresia. Otolaryngol Head Neck Surg 1990;103:931-7.

14. Schoem SR. Transnasal endoscopic repair of choanal atresia: Why stent? Otolaryngol Head Neck Surg 2004;131:3626.

15. Van Den Abbeele T, Francois M, Narcy P. Transnasal endoscopy treatment of choanal atresia without prolonged stenting. Arch Otolaryngol Head Neck Surg 2002;128:936-40.

16. Mcleod IK, Brooks DB, Mair EA. Revision choanal atresia repair. Int J Pediatr Otorhinolaryngol 2003;67:517-24.

17. Holzmann D, Ruckstuhl M. Unilateral choanal atresia: surgical technique ad longterm results. J Laryngol Otol 2002;116:601-4.

18. Dedo $\mathrm{H}$. transnasal mucosal flag rotation technique for repair of posterior choanal atresia. Otolaryngol Head Neck Surg 2001;124:674-82.

19. Uzomefuna $\vee$, Fergal Glynn F, Al-Omari $B$, Hone S, Russell, J. Transnasal endoscopic repair of choanal atresia in a tertiary care centre: A review of outcomes. Int J Ped Otorhinolaryngol 2012; 76:613-17.

20. Bozkurt MK, Keles B, Azimov A, Ozturk $\mathrm{K}$, Arbag, $\mathrm{H}$. The use of adjunctive topical mitomycin in endoscopic congenital choanal atresia repair. Int J Ped Otorhinolaryngol 2010;74:733-36.

21. Llorente JL, López $F$, Morato $M$, Suárez V, Coca A, Suárez C. Endoscopic treatment of choanal atresia. Acta Otorrinolaringol Esp. 2013;64(6):389-95. 